\title{
Effects of different alpha-1 adrenoceptor blockers on proximal urethral function using in vivo isovolumetric pressure changes
}

\author{
Takanori YAMAGUCHI ${ }^{1}$, Masashi NAGANO ${ }^{2}$ and Yukio OSADA ${ }^{2}$ \\ ${ }^{1}$ Department of Urology, Fukuoka City Medical Center for Sick Children and Infectious \\ diseases, 2-5-1 Tohïnmachi, Chuou-ku, Fukuoka 810-0063, Japan \\ ${ }^{2}$ Department of Urology, Miyazaki University School of Medicine, Miyazaki, Japan
}

\begin{abstract}
The effects of different alpha-1 adrenoceptor blockers on the urethra and the cardiovascular system were evaluated using an in vivo isovolumetric intra-urethral pressure model in New Zealand white rabbits. The urethra of anesthetized male rabbits was cannulated through the bladder and secured at the vesico-urethral junction. The distal side of urethra under the pubic bone was also closed to allow measurement of the intra-urethral pressure. Both the intra-urethral pressure and the femoral arterial pressure were monitored. The effects of five different alpha- 1 adrenoceptor blockers on the increases in both the intra-urethral pressure and blood pressure induced by phenylephrine were then examined. The inhibition rate of the alpha- 1 adrenoceptor blockers prazosin, bunazosin, terazosin, alfuzosin and tamsulosin on the increase in intraurethral pressure caused as a result of contraction by phenylephrine was $87.5 \pm 4.5 \%$ (mean \pm S.E.), $88.0 \pm 7.2 \%, 86.2 \pm 6.2 \%, 81.4 \pm 4.8 \%$ and $92.5 \pm 5.0 \%$ respectively. The potency ranking of these alpha-1 adrenoceptor blockers was tamsulosin $>$ bunazosin $>$ prazosin $>$ terazosin $>$ alfuzosin. Their inhibition rate of the arterial pressure increase induced by phenylephrine was $81.9 \pm 5.0 \%, 86.2 \pm 5.9 \%, 76.0 \pm 6.0 \%, 63.6 \pm 5.7 \%$ and $58.0 \pm$ $5.2 \%$ respectively, with a potency ranking of bunazosin $>$ prazosin $>$ terazosin $>$ alfuzosin $>$ tamsulosin. We therefore conclude that the alpha- 1 adrenoceptor blockers bunazosin and prazosin have a more potent action on both the urethra and the vascular system. However, tamsulosin and alfuzosin displayed a marked blockade of the increased urethral pressure induced by phenylephrine, with much less of a blockade of arterial pressure. In the present study, tamsulosin has been shown to be the most sensitive and powerful of the alpha- 1 adrenoceptor blockers on urethral smooth muscle.
\end{abstract}

Key words: alpha-1 adrenoceptor blockers, proximal urethra, in vivo model, isovolumetric intra-urethral pressure, blood pressure

Correspondence to: Takanori Yamaguchi, Ph.D., Fukuoka City Medical Center for Sick Children and Infectious Diseases, 2-5-1 Tohjinmachi, Chuou-ku, Fukuoka 810-0063, Japan

Phone: +81-92-713-3111 Fax: +81-92-713-3122 e-mail: yamaguchi.t@fukuoka-child.jp 


\section{Introduction}

Recent studies have shown that alpha-1 adrenoceptors are involved in the regulation of the contraction and relaxation of the smooth muscle of the proximal urethra and prostate gland. Basic and clinical studies have also shown that alpha-1 adrenoceptor blockers are effective in the release of tension in the proximal urethra and prostate gland, therefore reducing restriction of urinary flow. Since Caine et al. (1976) first reported the use of an alpha-1 adrenoceptor blocker to reduce the difficulty of urination caused by prostate gland hypertrophy, several other investigators have confirmed the usefulness of alpha-1 adrenoceptor blockers in the relief of this form of urinary obstruction (Sullivan et al., 1999; Pool et al., 2001; Thiyagarajan et al., 2002). Thus alpha-1 adrenoceptor blockers are now one of the best modalities for treatment of prostatic hypertrophy (AUA Practice Guideline Committee, 2003). However, alpha-1 adrenoceptor blockers, including prazosin as a typical example, are hypotensive drugs with side effects such as orthostatic hypotension. Although a number of different alpha-1 adrenoceptor blockers have been proved to be useful in placebo-controlled double blind studies and subsequently utilized for the treatment of prostatic hypertrophy, there have been few comparative studies on the effectiveness of these drugs. Therefore, in this study we have conducted a comparative study of various alpha-1 adrenoceptor blockers with regard to their selectivity and relative potency on the in vivo proximal urethra in an animal model. We have previously reported on the usefulness of this animal model for such studies (Yamaguchi et al., 1993).

\section{Materials and Methods}

Thirty-two New Zealand white male rabbits (weighing 2.0-2.5 kg, and about 6 months old) were anesthetized with an intravenous injection of pentobarbital $(25 \mathrm{mg} / \mathrm{kg})$. Each animal was opened through a midline incision over the lower abdomen. After resection of the pubic bone, the urethra was exposed. The distal end of urethra was ligated and a French size 5 catheter (outer diameter $1.7 \mathrm{~mm}$, inner diameter $0.7 \mathrm{~mm}$ ) inserted into the urethra from the bladder side and then secured at the vesico-urethral junction. The closed urethra was then filled with about $0.5 \mathrm{ml}$ of physiological saline. The pressure within the closed urethra was maintained at 40-50 $\mathrm{cm} \mathrm{H}_{2} 0$. Changes in the intra-urethral pressure as well as the blood pressure in the femoral artery were monitored simultaneously and recorded with a pressure recorder (12M-25, Nihon Koden). All autonomic drugs were administered via the auricular vein.

The urethra was contracted in these experiments using the alpha- 1 adrenoceptor stimulant phenylephrine. From a dose response curve (see Fig. 1), it was determined that the increase in intra-urethral pressure produced by injection of $5 \mu \mathrm{g} / \mathrm{kg}$ phenylephrine would provide an adequate control contraction of the urethral smooth muscle. The effect of each of the five different alpha- 1 adrenoceptor blockers tested was determined over a range of concentrations $(0.5,1,5,10,50 \mu \mathrm{g} / \mathrm{kg}$ respectively). This was expressed as the percentage inhibition the injection of the test drug had on the increase in intra-urethral pressure produced by the injection of $5 \mu \mathrm{g} / \mathrm{kg}$ phenylephrine following the injection of each concentration.

To compare the relative potency of three of the alpha- 1 adrenoceptor blockers (prazosin, 


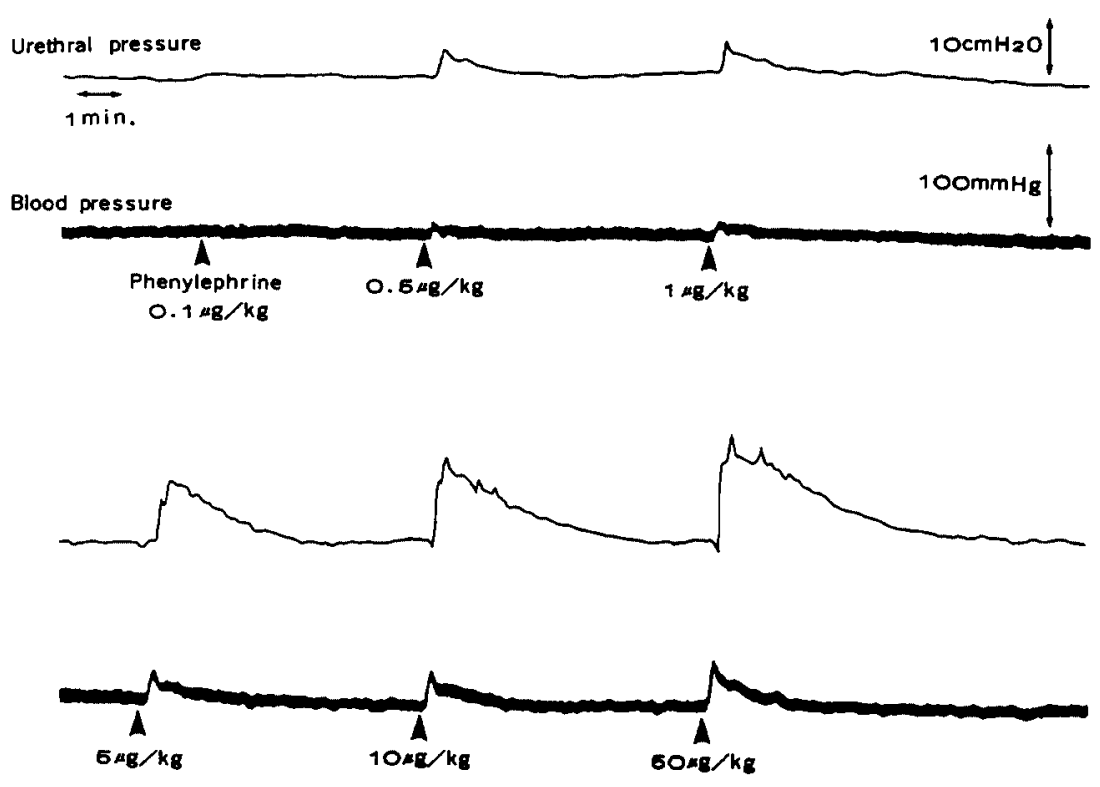

Fig. 1. Effects of the injection of increasing concentrations of the alpha-1 adrenoceptor stimulant phenylephrine on both the intra-urethral pressure and blood pressure in the in vivo isovolumetric intra-urethral pressure model in New Zealand white rabbits.

alfuzosin and tamsulosin) on the in vivo proximal urethra, each of these alpha-1 adrenoceptor blockers was injected at an increasing concentration from 0.1 to $50 \mu \mathrm{g} / \mathrm{kg}$. Following each drug injection a blood sample was taken from the femoral artery. Plasma concentrations of the particular alpha-1 adrenoceptor blocker in the unchanged form were measured with highpressure liquid chromatography.

Autonomic drugs used in this study were phenylephrine hydrochloride (Wako) as an alpha1 adrenoceptor stimulant, and prazosin hydrochloride (Fujisawa), alfuzosin hydrochloride (Fujisawa), terazosin hydrochloride (Mitsubishi), bunazosin hydrochloride (Ehsai) and tamsulosin hydrochloride (Yamanouchi) as alpha-1 adrenoceptor blockers.

\section{Results}

Following intravenous injection of phenylephrine, an alpha-1 adrenoceptor stimulant, both intra-urethral pressure and blood pressure increased dose-dependently (Fig. 1). As shown in Fig. 2, the intra-urethral pressure and blood pressure reached their peak values at $50 \mu \mathrm{g} / \mathrm{kg}$ of phenylephrine. The maximum value of the intra-urethral pressure was $42.7 \pm 1.8 \mathrm{~cm} \mathrm{H}_{2} \mathrm{O}$, while that of the blood pressure was $60.4 \pm 9.6 \mathrm{mmHg}$ (mean \pm S.E.) $(\mathrm{n}=6)$.

All of the five alpha-1 adrenoceptor blockers synchronously inhibited both urethral contraction and the increase in blood pressure induced by phenylephrine. The maximum inhibition rates of the intra-urethral pressure were $87.5 \pm 4.5 \%, 88.0 \pm 7.2 \%, 86.2 \pm 6.2 \%, 81.4 \pm$ $4.8 \%$ and $92.5 \pm 5.0 \%$ for prazosin, bunazosin, terazosin, alfuzosin and tamsulosin, respectively 

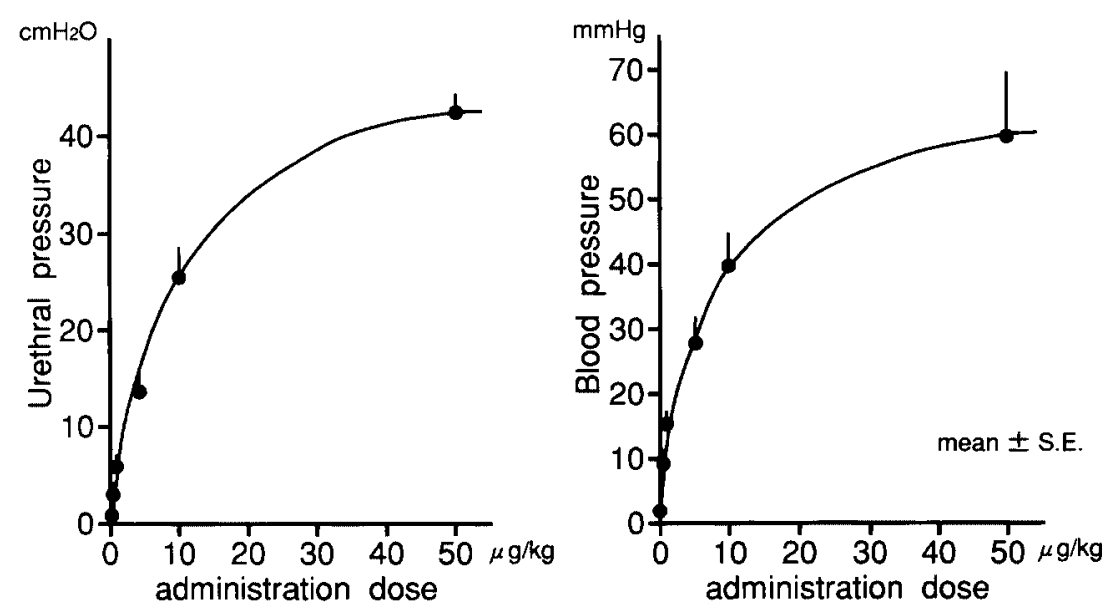

Fig. 2. Dose-response curves for the effects of phenylephrine on both intra-urethral pressure and blood pressure in the in vivo isovolumetric intra-urethral pressure model in New Zealand white rabbits.

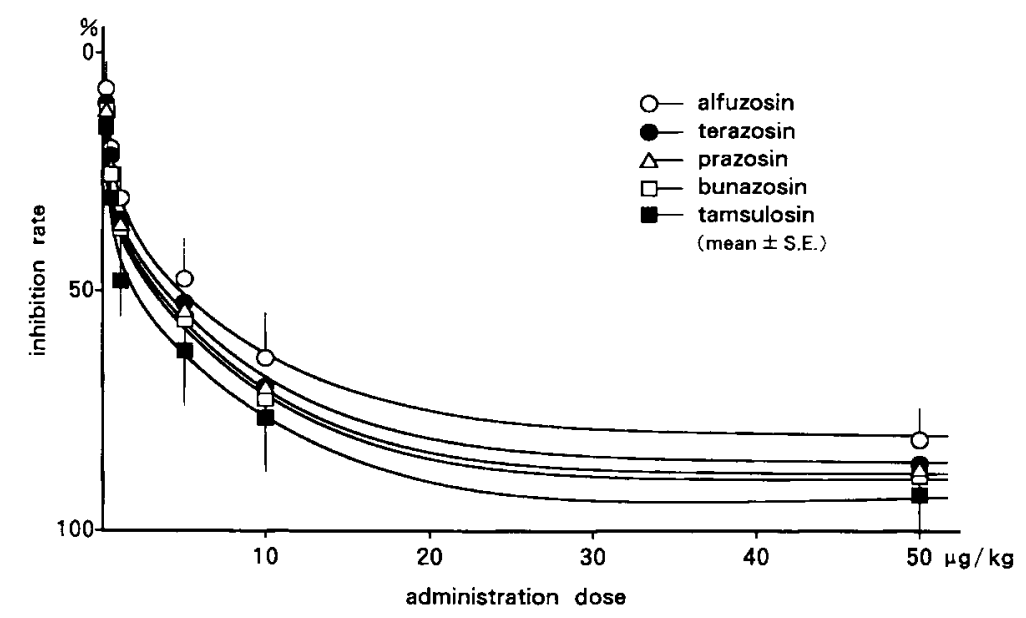

Fig. 3. Effects of alpha-1 adrenoceptor blockers on phenylephrine $(5 \mu \mathrm{g} / \mathrm{kg})$ induced increases in intra-urethral pressure in the in vivo isovolumetric intra-urethral pressure model in New Zealand white rabbits.

(Fig. 3). Although there were no significant differences between these values, the inhibition rate was highest for tamsulosin, followed by bunazosin, prazosin, terazosin and alfuzosin.

The inhibitory effect of each alpha-1 adrenoceptor blocker on the increase in blood pressure induced by $5 \mu \mathrm{g} / \mathrm{kg}$ of phenylephrine is shown in Fig. 4. There were significant differences between the maximum inhibition rates of prazosin $(81.9 \pm 5.0 \%)$, bunazosin $(86.2 \pm 5.9 \%)$, terazosin $(76.0 \pm 6.0 \%)$, alfuzosin $(63.6 \pm 5.7 \%)$ and tamsulosin $(58.0 \pm 5.2 \%)$. Prazosin and bunazosin showed the highest inhibition rate against the increase in blood pressure, followed by terazosin, alfuzosin and tamsulosin. Tamsulosin showed a significantly lower inhibition rate 


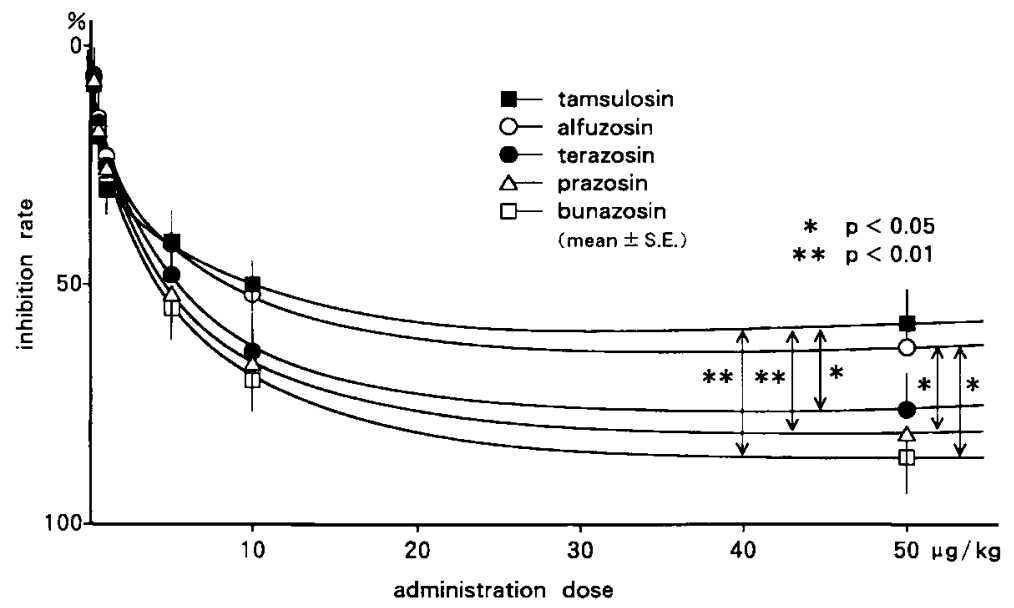

Fig. 4. Effects of alpha- 1 adrenoceptor blockers on phenylephrine (5 $\mu \mathrm{g} / \mathrm{kg})$ induced increases in blood pressure in the in vivo isovolumetric intra-urethral pressure model in New Zealand white rabbits.

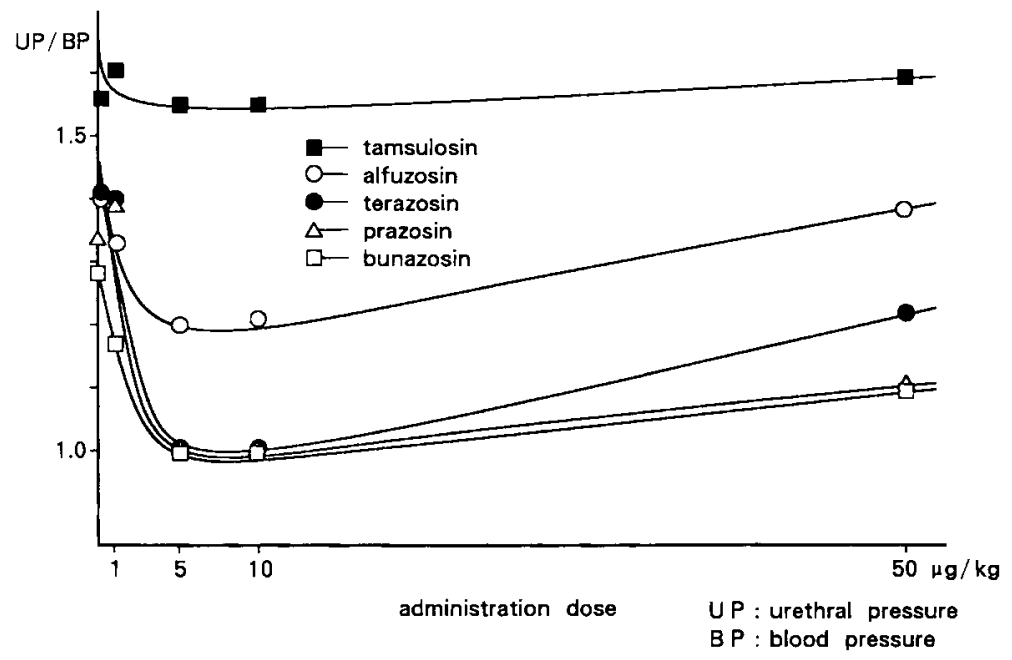

Fig. 5. Changes in the ratio of the inhibition rates (UP/BP) for different concentrations of each of the 5 alpha- 1 adrenoceptor blockers tested in the in vivo isovolumetric intraurethral pressure model in New Zealand white rabbits.

against the increase in blood pressure compared with the other alpha-1 adrenoceptor blockers.

To compare the specificity of each alpha-1 adrenoceptor blocker to the proximal urethra, an inhibition index against the percentage increase in intra-urethral pressure was calculated by defining the inhibition rate against the increase in blood pressure at each concentration as an index 1.0 (Fig. 5). A larger index would indicate a higher specificity for the proximal urethra. The index was 1.0-1.1 for both prazosin and bunazosin, while terazosin had a slightly higher index than these two drugs. Alfuzosin had an index of 1.2-1.3. For tamsulosin, the index was 


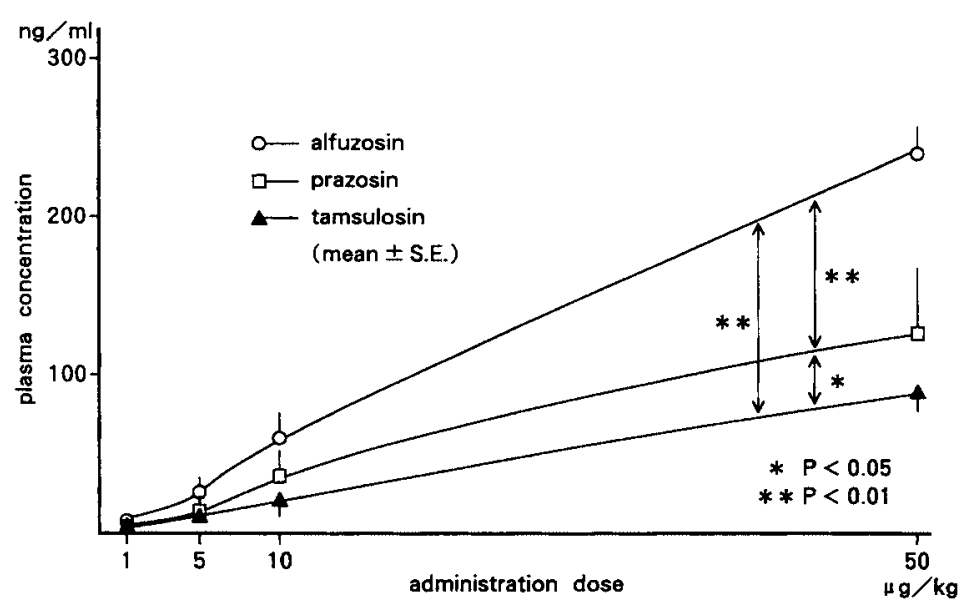

Fig. 6. Plasma concentrations of the 5 different alpha- 1 adrenoceptor blockers administered by injection at different concentrations in New Zealand white rabbits. Measurements made by high pressure liquid chromatography.

above 1.5 at all concentrations and thus demonstrated the highest specificity for the proximal urethra.

In a comparison of the plasma levels of prazosin, alfuzosin and tamsulosin, there were no significant differences at lower concentrations (Fig. 6). However, at higher concentrations, remarkable differences were found between them. At a concentration of $50 \mu \mathrm{g} / \mathrm{kg}$, the plasma level was $241.7 \pm 12.6 \mathrm{ng} / \mathrm{ml}$ for alfuzosin, $125.9 \pm 38.3 \mathrm{ng} / \mathrm{ml}$ for prazosin and $87.3 \pm 7.5 \mathrm{ng} / \mathrm{ml}$ for tamsulosin.

To compare the relative potencies between prazosin, alfuzosin and tamsulosin, a potential index was calculated by dividing either the maximum inhibition rate against phenylephrineinduced urethral contraction, or the increase in blood pressure due to phenylephrine, by the plasma level of each drug. The index was 0.7 against urethral contraction and 0.65 against the increase in blood pressure for prazosin. For alfuzosin, the index was 0.35 against urethral contraction and 0.26 against the increase in blood pressure. Alfuzosin had less than half the potency of prazosin against the increase in blood pressure. For tamsulosin, the index against the increase in blood pressure was similar to that of prazosin, while the index against urethral contraction was the largest of the three drugs tested (Fig. 7).

\section{Discussion}

Previously, the effects of alpha adrenoceptors have been studied mainly in the central nervous system and the cardiovascular system (references). Recent studies have shown that there is a significant population of post-synaptic alpha adrenoceptors in the lower urinary tract, and that these receptors play an important role in lower urinary tract function (Andersson $e t a l$, 1984; Kondo et al., 1993).

We have previously reported that both alpha- 1 and alpha- 2 adrenoceptors affect the 


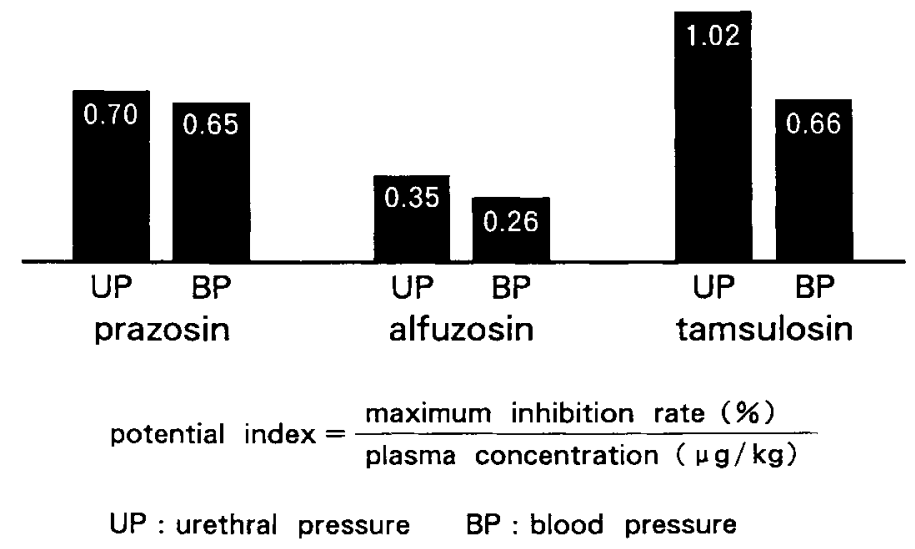

Fig. 7. Potential index of the five different alpha-1 adrenoceptor blockers in the in vivo isovolumetric intra-urethral pressure model in New Zealand white rabbits.

contraction of the proximal urethra in rabbits by using an in vivo isovolumetric intra-urethral pressure model (Yamaguchi et al., 1993). This animal model was used in the present study. Our previous results have also shown that there was a sex-related difference in the distribution of alpha-1 adrenoceptors in the rabbit proximal urethra. As alpha-1 adrenoceptors were more predominant than alpha- 2 adrenoceptors in male rabbits, they were used in this study. A study involving isometric contraction of isolated muscle strips of the human proximal urethra and prostate has revealed that alpha-1 adrenoceptors participate in contraction of the lower urinary tract in humans (Kunisawa et al., 1985).

With prostatic hypertrophy, the associated difficulty in urinating has been partly attributed to an increased urethral resistance elicited via alpha adrenoceptors (Caine et al., 1976; Sullivan et al., 1999; Pool et al., 2001; Thiyagarajan et al., 2002). This is supported by the facts that sections of the human prostate gland contract in vitro in response to norepinephrine and that the intra-urethral pressure at the level of the prostate gland also elevates on administration of norepinephrine. Phenoxybenzamine, the first drug utilized for the treatment of the urination difficulties resulting from prostatic hypertrophy, proved to be effective in inhibiting the contractile effect of norepinephrine (Lepor, 1993). However, since this drug had various side effects, such as vertigo, hypotension, palpitation and retrograde ejaculation, it was not widely used. Moreover, as phenoxybenzamine was suggested to be carcinogenic in rats, further development of the drug was abandoned. As a result of investigations concerning the subtype of alpha adrenoceptor that was involved in the human urinary tract, prazosin and other selective alpha-1 adrenoceptor blockers were developed and proved to be clinically useful (DeReijke $e t$ al., 2000; Chon et al., 1999). Prazosin, bunazosin and terazosin were first developed as hypotensive drugs and then additionally approved as drugs to alleviate the urination difficulties associated with the hypertrophy of the prostate gland. These drugs have the effect of eliciting orthostatic hypotension in vascular smooth muscle, as well as the effect of relaxing proximal urethral smooth muscle to relieve the difficulty of urinating. Alfuzosin and tamsulosin are new alpha-1 adrenoceptor blockers that have been developed to achieve selective effects on the 
proximal urethra and a lesser effect on blood pressure.

We have also investigated the specificity and the magnitude of potency of five different alpha-1 adrenoceptor blockers in the present in vivo study. Prazosin and bunazosin, which are both quinazoline derivatives, had remarkable inhibitory effects on both intra-urethral contraction and increased blood pressure. Terazosin, on the other hand, had a similar maximum inhibition rate against intra-urethral contraction and a lower inhibition rate against the increase in blood pressure, as compared with prazosin and bunazosin. But in the case of alfuzosin, there was a significant difference between the inhibition rates against urethral contraction (81.4\%) and against the increase in blood pressure (63.6\%). It is therefore suggested that among the quinazoline derivatives tested, alfuzosin is the most suitable alpha- 1 adrenoceptor blocker for the treatment of urination difficulty. Tamsulosin, classified as a sulfamoyl phenethylamine, has a different structure to the quinazoline derivatives. The maximum inhibition rate against urethral contraction was $92.5 \%$ for tamsulosin, higher than for bunazosin or prazosin, while the maximum inhibition rate against an increase in blood pressure was a remarkably low $58.0 \%$. Thus tamsulosin is the most effective alpha- 1 adrenoceptor blocker for the relief of proximal urethral contraction and has the mildest effects on blood pressure of the drugs tested in the present study.

To compare the in vivo potency of the drugs, we measured the plasma levels of each of the alpha-1 adrenoceptor blockers. There were unexpectedly significant differences between the plasma levels of prazosin, alfuzosin and tamsulosin at higher concentrations, although these differences were not evident at the lower concentrations. We speculate that these significant differences are the result of differences in circulatory dynamics and the rates of metabolism of each of the drugs. In a clinical setting, administration of prazosin at a concentration of $2 \mathrm{mg}$ results in a maximum plasma concentration of prazosin of about $20 \mathrm{ng} / \mathrm{ml}$ (Hayashi et al., 1987). According to the dose-concentration curve shown in Fig. 6, this concentration corresponds to the concentration induced by injection of $5 \mu \mathrm{g} / \mathrm{kg}$ prazosin in the present study. Therefore, it is reasonable to compare effects of alpha- 1 blockers at a concentration of $5 \mu \mathrm{g} / \mathrm{kg}$. The dose-effect curves indicate the same above-mentioned significant differences between the plasma levels of the different alpha- 1 adrenoceptor blockers at a concentration of $5 \mu \mathrm{g} / \mathrm{kg}$.

Differences in specificity between alpha-1 adrenoceptor blockers can probably be attributed to different subtypes of alpha-1 adrenoceptor receptors. Alpha-1 adrenoceptors have been classified into the subtypes of alpha-1A, alpha-1B and alpha-1D depending on the receptor protein (Ford et al., 1994). The alpha-1 adrenoceptor genes formerly known as alpha-1 A/D, alpha-1B and alpha-1C were renamed alpha-1D, alpha-1B and alpha-1A, respectively. Lepor et al. (1995) reported that alpha-1A adrenoceptors were located in the prostate gland of the rabbit and rat, alpha-1A and alpha-1D adrenoceptors were located in the human prostate gland, and alpha$1 \mathrm{~B}$ adrenoceptors were located in the human vasculature in receptor binding experiments with extirpated organs. Price et al. (1993) also suggested that alpha-1D adrenoceptors are most predominantly distributed in the human prostate gland and the detrusor muscle in a study on prostatic alpha-1 adrenoceptors using molecular biology studies.

A recent investigation suggested that although prazosin shows no specificity to any subtype of alpha-1 adrenoceptors, tamsulosin has a remarkable affinity to alpha-1A and alpha-1D 
adrenoceptors. This affinity to alpha-1 A/D adrenoceptors contributes to the selective effects of tamsulosin in the relief of urination difficulties resulting from prostate gland hypertrophy. However, many problems remain regarding the classification of alpha-1 adrenoceptor subtypes and further investigations are required.

Future molecular biological studies on specific drugs for alpha-1 adrenoceptors in the lower urinary tract will reveal the difference between the alpha- 1 adrenoceptors located in the lower urinary tract and those in the cardiovascular system. It is expected that more specific drugs for treatment of lower urinary diseases, such as prostate gland hypertrophy, will be developed.

\section{References}

AUA Practice Guideline Committee (2003). AUA guideline of management of benign prostatic hyperplasia. Chapter 1: Diagnosis and treatment recommendations. J. Urol. 170: 530-547.

Andersson, K.E., Larsson, B. and Sjogren, C. (1984). Characterization of the alpha-adrenoceptors in the female rabbit urethra. Br. J. Pharmacol. 81: 298-300.

Caine, M., Pfau, A. and Perlberg, S. (1976). The use of alpha-adrenergic blockers in benign prostatic obstruction. Br. J. Urol. 48: 255-263.

Chon, J.K., Borkowski, A., Partin, A.W., Isaacs J.T., Jacobs, S.C. and Kyprianou, N. (1999). $\alpha 1$ Adrenoceptor antagonists terazosin and doxazosin induced prostate apoptosis without affecting cell proliferation in patients with benign prostatic hyperplasia. J. Urol. 161: 2002-2008.

DeReijke, T.M. and Klarskov, P. (2000). Doxazosin versus alfuzosin in benign prostatic hyperplasia: results of a multinational, randomized, double-blind European trial. Eur. J. Urol. 37: 473-479.

Ford, A.P., Williams, T.J., Blue, D.R. and Clarke, D.E. (1994). Alpha 1-adrenoceptor classification: sharpening occam's razor. Trends. Pharmacol. Sci. 15: 167-170.

Hayashi, M., Hanano, M. and Shintani, F. (1987). Relative bioavailability of prazosin hydrochloride tablet in man. Jpn. J. Clin. Pharmacol. 9: 385-394.

Kunisawa, Y., Kawabe, K., Niijima, T., Honda, K. and Takenaka, T. (1985). A pharmacological study of alpha adrenergic receptor subtypes in smooth muscle of human urinary bladder base and prostatic urethra. J. Urol. 134: 396-398.

Lee, E. and Lee, C. (1997). Clinical comparison of selective and nonselective alpha 1A-adrenoreceptor antagonists in benign prostatic hyperplasia: studies on tamsulosin in a fixed dose and terazosin in increasing doses. Br. J. Urol. 80: 606-610.

Lepor, H., Tang, R., Kobayashi, S., Shapiro, E., Forray, C., Wetzel, J.M. and Gluchowski, C. (1995). Localization of the $\alpha 1 \mathrm{~A}$-adrenoceptor in the human prostate. J. Urol. 154: 2096-2099.

Lepor, H. (1993). Medical therapy for benign prostatic hyperplasia. Urol. 42: 483-501.

Lomasney, J.W., Cotecchia, S. and Lefknowitz, R.J. (1987). Molecular biology $\alpha$-adrenergic receptors; implications for receptor classification and structure-function relationship. Biochim. Biophys. Acta. 127: 1095-1099.

Pool, J.L. and Kirby, R.S. (2001). Clinical significance of alpha 1-adrenoceptor selectivity in the management of benign prostatic hyperplasia. Int. Urol. Nephrol. 33: 407-412.

Price, D.T., Schwinn, D.A., Lomasney, J.W., Allen, L.F., Caron, M.G. and Lefkowitz, R.J. (1993). Identification, quantification, and localization of mRNA for three distinct alpha1 adrenergic receptor subtypes in human prostate. J. Urol. 150: 546-551.

Sullivan, J. and Abrams, P. (1999). Alpha-adrenoceptor antagonists in neurogenic lower urinary tract dysfunction. Urol. 53 (Suppl 3A): 21-28.

Thiyagarajan, M. (2002). Alpha-adrenoceptor antagonists in the treatment of benign prostate hyperplasia. Pharmacol. 65: 119-124. 
Yamaguchi, T., Kitada, S. and Osada, Y. (1993). The role of adrenoceptors in the proximal urethral function of female and male rabbits using an in vivo model of isovolumetric urethral pressure generation. Neurourol. Urodyn. 12: 49-57.

(Received July 25, 2005; Accepted August 26, 2005) 